A N N A L E S Annales de Bretagne et des Pays de l'Ouest

Anjou. Maine. Poitou-Charente. Touraine

124-1 | 2017

Varia

\title{
Histoire de Redon. De l'abbaye à la ville
}

\section{Olivier Charles}

\section{OpenEdition}

Journals

Édition électronique

URL : http://journals.openedition.org/abpo/3516

DOI : $10.4000 / a b p o .3516$

ISBN : 978-2-7535-5677-5

ISSN : 2108-6443

Éditeur

Presses universitaires de Rennes

Édition imprimée

Date de publication : 30 avril 2017

Pagination : 165-168

ISBN : 978-2-7535-5675-1

ISSN : 0399-0826

Référence électronique

Olivier Charles, "Histoire de Redon. De l'abbaye à la ville », Annales de Bretagne et des Pays de l'Ouest

[En ligne], 124-1 | 2017, mis en ligne le 30 avril 2017, consulté le 23 septembre 2020. URL : http:// journals.openedition.org/abpo/3516; DOI : https://doi.org/10.4000/abpo.3516 


\section{Comptes rendus}

ArThuis, Rémy, Monteil, Martial (dir.), Archéologie de la Basse-Loire, Rennes, PUR, collection "Archéologie \& Culture ", 2015, 194 p.

Cet ouvrage, qui présente des études récentes consacrées à l'archéologie de la Loire entre Ingrandes et Couëron, résulte d'un Projet Collectif de Recherche intitulé " des Ponts-de-Cé à l'estuaire ", né en 2006-2007 qui visait à coordonner diverses actions bénévoles, professionnelles (INRAP) ou universitaires (Université de Nantes). Les deux coordonnateurs de la publication, Rémy Artuis, géomorphologue à l'INRAP, et Martial Monteil, actuellement professeur à l'université de Nantes, soulignent la difficulté qu'il y a à synthétiser des travaux menés à des échelles, par des équipes, en des lieux et avec des moyens extrêmement différents. À cela s'ajoutent encore les grandes différences d'approche, de chronologie, de regard et de vocabulaire entre la chercheuse en archives, le fouilleur en rives et lit de Loire, et le géomorphologue. Il en résulte un ensemble de cinq contributions juxtaposées et bien distinctes, qui reflètent la labilité, la variabilité, voire l'insaisissabilité historique, archéologique et juridique du fleuve Loire, que l'homme n'a jamais réussi à maîtriser.

La première contribution de Rémy Artuis et Jean-François Nauleau s'inscrit dans la suite de ce que Kerviler dénomma " chronomètre préhistorique " dans sa publication des travaux de Penhouët. Ici l'étude tente de synthétiser l'apport de 1000 sondages géotechniques, en leur adjoignant les données des fouilles menées en quelques lieux comme Rezé, et en affinant la seule stratigraphie par des datations ${ }^{14} \mathrm{C}$. Ainsi ont pu être cartographiées les grandes phases de dépôt sur le substrat profond, nommé " le rocher de Nantes ", au cours des 10000 ans qui nous ont précédés. Comblement rapide d'une vallée fluviale d'abord en réseaux tressés puis à cours unique au fur et à mesure de la remontée du niveau de la mer, formation d'une ria tentaculaire ennoyant les basses vallées de la Loire, de l'Erdre, de la Sèvre, jusqu'à Oudon, et fixation d'un niveau marin proche de celui d'aujourd'hui vers 5700 BP. Commence alors un colmatage atteignant parfois 15 mètres, fait de vases marines et de sables fluviatiles, néanmoins marqué par des reprises d'érosion lors d'abaissements temporaires du niveau marin. Pour les périodes antiques et médiévales, les géomorphologues constatent la stérilité d'une confrontation de leurs données à celles de l'archéologie, tant les deux approches diffèrent en termes d'échelle spatiale. En conclusion, ils avouent que leur étude géologique et géomorphologique de la Basse Loire pendant l'Holocène révèle une histoire très complexe, dans laquelle les données pointues de leur recherche peinent encore à éclairer l'action de l'homme sur le fleuve, tant celui-ci l'emporte par sa puissance.

Yann Viau et Véronique Guitton, présentent ensuite un ensemble de découvertes archéologiques faites en rive de Loire au Marillais, à deux pas de Saint-Florent-leVieil. On doit à des bénévoles la première connaissance de ce site placé au confluent de l'Ėvre et de la Loire, entre autres grâce à la découverte d'importants vestiges de céramique gallo-romaine. Ces preuves d'une occupation ancienne, ajoutées au voi- 
sinage immédiat du très important monastère du Mont Glonne, ont amené Y. Viau à mener trois campagnes de fouille lors des étés 2006, 2007 et 2008. Chacune d'elles a permis de mettre au jour une grande quantité de vestiges de bois presque tous liés à des aménagements et machines hydrauliques. Les plus importants appartiennent à un ou des moulins à eau, tels des éléments de rouets comme des alluchons, des pièces de roue à aube dont une archéologiquement complète, mais aussi des planches de vannes, une nasse à poissons, des poteaux, une possible roue de brouette, et même un vase de bois. L'analyse de 259 de ces restes et la datation ${ }^{14} \mathrm{C}$ de plusieurs d'entre eux, ont montré que la plupart étaient réalisés en chêne ou en frêne, et qu'ils étaient liés à un ou des moulins tout proches qui fonctionnèrent du $\mathrm{VII}^{\mathrm{e}}$ au $\mathrm{X}^{\mathrm{e}}$ siècle, juste en amont de la zone fouillée. On ne soulignera jamais assez la rareté et l'importance de telles découvertes car elles révèlent la technologie du moulin à eau pour une période qualifiée naguère d'obscure. Les auteurs constatent là encore la grande difficulté qu'il y a à mettre en relation ces données matérielles avec les données écrites, même si l'on sait que l'abbaye Saint-Florent-le-Viel sur le Mont Glonne existait à cette époque, et qu'une longue occupation normande est quasi-attestée sur l'Île Batailleuse, au pied du monastère. Certes, il faut tenter de confronter ces deux types de sources, mais c'est aussi l'occasion de dire que le temps où l'on pensait que l'histoire ne pouvait se fonder que sur les sources écrites est maintenant dépassé, et que l'archéologie permet tout autant de nourrir la connaissance et la synthèse historique.

Tout près des Marillais, au pied du mont Glonne, d'autres aménagements hydrauliques anciens ont été détectés et ils font l'objet d'une troisième contribution, celle de Denis Fillon. Nombre d'entre eux ont été repérés dans les sources écrites du XIV ${ }^{e}$ au XVII ${ }^{e}$ siècle, mais et il reste encore à étudier les précieux cartulaires de Saint-Florent pour en rechercher des mentions plus anciennes. Grâce à la fréquence et à la durée sans cesse croissantes des étiages de la Loire, il a d'abord été possible de procéder à des sondages subaquatiques, en particulier au "Gué au moine ", au pied même du monastère. Ensuite il a pu être procédé à un lever topographique et à un sondage, ce qui a mis en évidence une levée empierrée, large de $20 \mathrm{~m}$ sur une longueur de $60 \mathrm{~m}$, qui semble bien confirmer l'existence ancienne d'un franchissement aménagé, utilisable lors des basses eaux. Tout aussi intéressants et nouveaux, dans ce même secteur, des systèmes de pieux clayonnés disposés en V multiples et accolés ont aussi pu être relevés. Ils constituaient autant de systèmes destinés à contraindre et mener anguilles, saumons, aloses et esturgeons vers des nasses disposées en amont et en aval. Les datations ${ }^{14} \mathrm{C}$ réalisées sur quelques pieux se placent toutes au Moyen Âge central, mais en raison des multiples réfections nécessaires sur ces installations, l'archéologue pense que le système peut être antérieur. Au total, les nombreuses observations, et surtout les opérations de 2015, auront permis d'accumuler un grand nombre de données. En leur état actuel, encore très partiel, elles peuvent paraître compliquées, voire confuses. Elles ont néanmoins le grand mérite de décrire la nature et la complexité des aménagements halieutiques sur la Loire près de Saint Florent au Moyen Âge, leur évolution constante, et surtout leur densité. Il est à souhaiter qu'elles puissent être poursuivies. Là encore, si l'on s'en tenait aux seules sources écrites médiévales, qui visaient avant tout à établir des droits et des revenus seigneuriaux, on ignorerait tout de la réalité matérielle de ces pêcheries.

Dans ce recueil à dominante géologique, géomorphologique et archéologique, une contribution, celle d'Emmanuelle Miéjac, ouvre cependant une autre fenêtre et donne un autre regard, ceux d'une approche de la Loire au travers des données d'archives. Elle traite des informations liées au fleuve et à l'eau dans les sources de l'abbaye Saint Florent. Après un rappel de l'histoire de l'abbaye, intimement liée 
au fleuve, ainsi que des riches fonds conservés aux Archives départementales de Maine-et-Loire, l'auteur livre un intéressant début d'inventaire des ressources tirées de l'eau et du fleuve par les moines, de leurs droits de péage, port et passage, de leurs moulins, étangs et pêcheries sur la Loire et sur l'Ėvre, depuis le xII jusqu'au XVIII ${ }^{\mathrm{e}}$ siècle. Elle rappelle aussi que ce sont les récentes découvertes faites sur l'Èvre au Marillais qui ont induit sa recherche. Aucun lien direct n'a cependant pu être établi entre ses découvertes et celles des archéologues. Une fois encore, on mesure la difficulté de l'exercice, celle qu'il y a à faire de la pluridisciplinarité plus qu'une simple formule, tant les deux recherches diffèrent par leur forme, leur fond, leur échelle, leur finalité. Il va de soi que les scribes de Saint-Florent du $\mathrm{XII}^{\mathrm{e}}$ siècle exprimaient avant tout la domination supra-terrestre du spirituel sur le temporel, bien loin des préoccupations infra-terrestres de l'archéologie au Xxle siècle. La conjonction ne peut qu'être très partielle, voire contradictoire, elle n'en est pas moins nécessaire car elle complète ou corrige le faisceau de l'enquête historique. Cependant, pour le cas, elle nécessiterait un filtrage total des sources de Saint-Florent, travail démesuré quand on sait que, même si au XIXe siècle P. Marchegay a édité plusieurs recueils de chartes de l'abbaye, aucun des cartulaires de celle-ci n'a encore été publié.

La dernière partie de l'ouvrage, fruit de la collaboration de Rémy Artuis, Emmanuelle Miéjac et Jean-François Nauleau, traite de la Boire Torse, un canal creusé entre Ingrandes et Anetz. Celle-ci se présente sous la forme d'une sorte de chenal large d'environ 20 mètres et long à l'origine de 15 kilomètres, mais réduit de moitié au XIX ${ }^{\mathrm{e}}$ siècle par la chaussée du chemin de fer. Originellement connecté à la Loire à ses deux extrémités, il suivait sur la rive droite le bord non inondable du fleuve, séparé de celui-ci par des terres basses dont la Grande Prée de Varades. L'étude se propose de répondre à la question de l'origine de cette structure, et de montrer si elle est naturelle ou anthropique. Plutôt que de rappeler la pauvre et tardive définition du Littré, il semble qu'il aurait été utile d'approfondir le sens originel de ce mot boire/bouère/boere très lié à la Loire et d'en rechercher les occurrences et le sens anciens : il figure dans la Coutume générale de Touraine, Rabelais en fait usage et on en a mention dès 1110-1130 pour Saint-Maur. Une " pré-étude " en archives, menée par E. Miéjac a d'abord permis de montrer que des cartes et surtout des documents écrits attestent, au moins dès 1609, l'existence de cette boire. Par la suite, celle-ci apparaît ici et là, large de 10 toises vers Montrelais. L'étude consiste surtout en 10 pages d'une lourde étude géomorphologique qui consista à réaliser 244 tranchées à la pelleteuse et à en tirer des conclusions. Il en ressort que la boire était un canal aménagé sur un ancien bras de la Loire, sur lequel, hormis le palissage d'un bras annexe, il n'a été fait aucune découverte archéologique. Seules des datations ${ }^{14} \mathrm{C}$ réalisées sur des strates organiques et sur des bois ont permis de dater l'ouvrage entre environ 800 et la fin du XII siècle. Quant à sa fonction, les auteurs concluent, mais sans argument décisif, qu'il était destiné à desservir les petites localités riveraines et permettre une navigation plus aisée que sur le fleuve, hors des périodes d'étiage. Beaucoup de questions demeurent pendantes.

Les responsables de la publication terminent l'ouvrage par six pages consacrées aux acquis et aux perspectives de cette recherche et de ce programme. Outre ce qui a été détaillé ci-dessus, ils insistent sur deux nécessités : celle de la prise en main d'une programmation de la recherche dans la fenêtre de Nantes et Rezé par le Service Régional de l'Archéologie des Pays de la Loire, et la nécessité de trouver plus de moyens pour soutenir le travail des bénévoles et les fouilles programmées dans la fenêtre d'Ancenis. Ils insistent à juste titre sur l'importance des découvertes faites au Gué au moine et plus encore au Marillais. On ne peut que plaider dans le même sens car il y a là, nous semble-t-il, un champ de recherche archéologique 
particulièrement neuf et dynamique, comme en témoigne la publication en 2016 du remarquable colloque de Lons-le-Saunier consacré aux moulins hydrauliques et à vent. Nombre de ces découvertes majeures portant sur le Moyen Âge, période aussi documentée par l'écrit que par l'archéologie, l'occasion est donnée de développer une réelle co-recherche entre les deux approches. Si l'on ajoute la création à l'université de Nantes d'un poste de maître de conférences en archéologie subaquatique, on peut espérer que les connaissances géo-archéologiques, archéologiques et archivistiques ne vont faire que s'enrichir, et contribuer plus encore à l'Histoire du Fleuve Loire

Jean-Claude MEuRET

GENDRY, Mickaël, De l'Armorique à la Bretagne. Les Bretons et l'Armorique au haut Moyen Âge, Paris, Éditions 7 Écrit, 2016, 410 p.

Le livre de Mickaël Gendry est souvent défectueux du point de vue de la forme : émaillé de nombreuses coquilles et cacographies, qu'il faut en conséquence renoncer à signaler, écrit dans un style dont il aurait fallu en maintes occasions polir l'expression, souvent redondant à force de redites, - ah, les dangers du copiécollé! -, l'ouvrage porte ainsi témoignage qu'il n'a malheureusement pas bénéficié des corrections et des critiques qu'aurait pu lui apporter un véritable comité de lecture. En outre, s'agissant de son utilisation par les chercheurs, il est regrettable de ne pouvoir bénéficier d'une bibliographie récapitulative des nombreuses références mentionnées dans les notes, ni d'un index, d'autant que renvois et citations se révèlent à l'occasion approximatifs. Quant au fond, $M$. Gendry ne s'approprie pas toujours nettement les résultats de ses propres découvertes et donne ainsi au lecteur l'impression d'un certain flou, qui n'est pas totalement dissipé par la rapide synthèse proposée en conclusion : cette ambiguïté est surtout manifeste pour ce qui concerne la situation politique, militaire et religieuse de la péninsule armoricaine à la fin du Bas-Empire.

Ces remarques, qui peuvent sembler sévères, sont au contraire destinées à mettre en évidence le fait que ce livre, qui témoigne de la passion et du travail important de son auteur, présente à nos yeux plusieurs mérites, au moins au nombre de trois : d'abord, la volonté de prendre en compte des acquis des quelque trente dernières années de recherches hagiologiques bretonnes; ensuite, la démonstration que l'histoire des origines de la Bretagne n'a pas été élaborée, ex nihilo, au IX siècle dans les écritoires monastiques locaux, en particulier à Landévennec, comme certains travaux récents pourraient donner à le penser; enfin, la tentative de reconstitution de l'histoire institutionnelle régionale, depuis la période du Bas-Empire jusqu'aux lendemains de la Réforme carolingienne, par le biais d'une interprétation " juridicisante " des sources, adaptée au demeurant à la nature de plusieurs d'entre elles. À cette occasion, au-delà des évêchés qui font l'objet d'un traitement approfondi, M. Gendry examine rapidement les autres entités ecclésiales bretonnes, "ploues et lan" ( $\mathrm{sic}$ ), et la façon dont leur réseau vient se combiner à celui des vici et des pagi (p. 229-242)

Proposer un état de la recherche sur " les origines de la Bretagne ", pour reprendre le titre de l'ouvrage séminal de Léon Fleuriot, paru en 1981, en tenant compte du changement de paradigme qui, depuis une trentaine d'années, caractérise les travaux sur l'hagiographie régionale, enfin définitivement libérée des chimères " laborderistes " et de leurs avatars, constitue un projet ambitieux qui, à lui seul, 
mériterait un volume : ainsi, malgré l'ampleur de leurs propos respectifs, dont la confrontation s'avère particulièrement enrichissante, le précieux Répertoire raisonné consacré en 2009 par Joseph Claude Poulin à L'hagiographie bretonne du haut Moyen Âge, et, en 2012, le livre-testament du regretté Bernard Merdrignac, D'une Bretagne à l'autre, dont nous avons rendu compte ici même (Annales de Bretagne et des Pays de l'Ouest, 119 (2012), n ${ }^{\circ}$ 4, p. 143-145), ouvrages qui sont abondamment cités par M. Gendry, n'ont pas épuisé le sujet. De surcroît, en 2011, une chercheuse britannique, Caroline Brett, dont le travail sur les migrations bretonnes dans la péninsule a été partiellement relayé en Bretagne par Magali Coumert, s'est efforcée de montrer, avec des arguments assez inégaux, que la majorité des chercheurs continentaux continuait, en l'occurrence, à travailler dans le cadre d'une doxa " fleuriotiste " devenue obsolète, ce qui avait pour conséquence de disqualifier largement les résultats de leurs travaux : s'est engagée à cette occasion, sur fond de controverse, mais avec un réel allant, une réflexion renouvelée sur ces questions. M. Gendry, sans doute un peu prématurément, croit venu le temps de la synthèse et, prenant son bien chez les uns comme chez les autres, - rejetant ainsi définitivement, à la suite de C. Brett et de M. Coumert, mais aussi de Patrick Galliou, l'hypothèse " fleuriotiste " d'une immigration bretonne précoce, de nature militaire, dans la presqu'île armoricaine (p. 17-18 et 49-57); ou bien reconnaissant, avec Patrice Lajoye, dans le personnage de Tugdual un évêque de Lisieux, dont le siège épiscopal aurait été doté au vi ${ }^{\mathrm{e}}$ siècle de biens du fisc à Tréguier et dans ses parages (p. 184-189), sans parler du possible rôle " structurant " joué à Quimper par son supposé minihi local (p. 309-323) -, il propose de manière assez crâne de confronter le matériau hagiographique, dont il ne méconnaît pas au demeurant le caractère particulier, à d'autres sources plus anciennes, de nature juridique, - textes canoniques (p. 175-183) et civils (p. 217-227) -, pour en extraire des indications séduisantes, sinon convaincantes, sur la façon dont l'établissement continental de populations bretonnes insulaires a pu s'effectuer, à l'époque mérovingienne, dans le cadre romano-franc. M. Gendry souligne ainsi (p. 16) que "l'approche méthodologique des mythes étiologiques de l'Antiquité peut être facilement adaptée aux vies des saints mettant en scène des rites de fondation [...]. La lecture en négatif des vies de saints (vitae) donne en effet à voir un mode de représentation et d'appropriation de l'espace, une "topographie du sacré" et le projet de leurs auteurs (les hagiographes et/ou leurs commanditaires) "; et de citer à ce sujet (p. 17) l'exemple " de la vita tardive de Méen (XII e siècle) dont le lien avec le diplôme d'immunité de Saint-Méen et de l'île de Malo a souligné combien elle était importante, aux côtés des autres vies de saint Malo de l'époque carolingienne ou des autres sources, y compris archéologiques, pour comprendre l'origine de l'évêché d'Alet ". Rappelons que la comparaison entre sources hagiographiques et sources normatives a fait récemment l'objet d'un colloque dont les actes, publiés en 2014, contiennent une communication de Claire Garault qui justement porte sur "Les rapports entre récits hagiographiques et matériel diplomatique à travers le dossier hagiographique de saint Malo (IXe-XII siècle)".

On peut lire en filigrane tout au long de son ouvrage la conviction qui soutient le propos de M. Gendry : empruntée à son maître B. Merdrignac, elle le rapproche davantage de Fleuriot et l'oppose en la circonstance à la stricte Quellenforschung à laquelle J.-C. Poulin limite ses précieuses investigations, ainsi qu'aux doutes exprimés par C. Brett ou par M. Coumert sur la possibilité de transmission par l'hagiographe d'un élément factuel issu d'un passé révolu. "Si le but des hagiographes n'était pas de faire de l'histoire ", écrit ainsi M. Gendry (p. 16), "ils n'étaient pas moins capables que nous à [sic] évoquer leur propre passé ". En effet, les efforts récents et souhaitables entrepris pour ramener le matériau hagiographique à la seule dimension de ses principales données littéraires, - répertoire de motifs folk- 
loriques, " mode d'emploi " de reliques, catalogue de droits et de privilèges -, ont-ils sans doute parfois fait perdre de vue que, pour la communauté qui, à un titre ou à un autre, à tort ou à raison, se reconnaissait tributaire du saint concerné, le texte hagiographique constituait avant tout, - si du moins l'on excepte bien sûr de parler de l'essentiel, c'est-à-dire la dimension spirituelle et édificatrice du propos -, un "récit des origines ", où se sont entrecroisés, indémêlables pour l'historien, les fils de la mémoire et de l'histoire; ainsi, préconiser que de tels " récits des origines" auraient pu naître de manière spontanée en Bretagne à l'époque carolingienne sous la plume des hagiographes, s'agissant notamment de ce qui se rapporte à une division territoriale en "royaumes ", qui figure déjà chez Grégoire de Tours, c'est délibérément privilégier la possibilité que la mémoire des migrations bretonnes ait été localement oblitérée, alors même qu'elle " était encore bel et bien vivace, un siècle et demi après, à la cour des souverains francs ", comme le souligne M. Gendry à propos d'un passage d'Eginhard (p. 75, n. 205) : la prudence consiste ici, non pas à se revendiquer de la pratique du rasoir d'Ockham, mais, bien au contraire, à garder largement ouvert l'éventail des hypothèses.

Cette position est manifestement celle de M. Gendry, quand il suppose que l'installation des Bretons sur le continent, dans le prolongement d'une possible initiative de Syagrius (p. 68-70), encore renforcée par Clovis (p. 75-84), a surtout été encouragée par Childebert, dans un contexte où ce dernier, - qui retrouve sous la plume de l'auteur, le rôle central que lui ont attribué en leur temps les hagiographes (p. 183-200) -, aurait ainsi profité du démantèlement de l'ancien Tractus armoricanus et nervicanus, pour opérer un transfert massif de terres publiques au profit de l'Église; transfert dont la contrepartie aurait consisté dans le contrôle des nouveaux sièges épiscopaux créés à cette époque, à savoir Dol et Saint-Pol-de-Léon (p. 135183). Cette conjecture, inédite et hardie, implique corollairement qu'à l'époque de la "normalisation carolingienne " qui, selon une hypothèse désormais reçue par la majorité de la communauté scientifique, a vu la fondation des sièges de Quimper et d'Alet, ce dernier évêché aurait été démembré de celui de Dol et non l'inverse (p. 175-176) : M. Gendry insiste (p. 384) sur le fait que les souverains francs ont, à nouveau, utilisé à cette occasion " les terres fiscales comme levier de leur politique ", cette fois " par l'octroi de diplômes d'immunité en bonne et due forme", comme il en fait la longue démonstration pour l'évêché d'Alet (p. 258-307); le cas de Quimper apparaît en revanche plus complexe, car le processus de normalisation que nous avons rappelé intervient ici sur fond de compétition hagio-politique entre la triade Gradlon, Corentin, Guénolé, et son prototype plus ancien, incarné par Tugdual (p. 323-376).

André-Yves BouRGÈs

PÉPIN, Guilhem, LaINÉ, Françoise, Boutoulle, Frédéric (éd.), Routiers et mercenaires pendant la guerre de Cent ans. Hommage à Jonathan Sumption, Bordeaux, Ausonius, coll. « Scriptoria Mediævalia; 28 ", 2016, 357 p.

Cet ouvrage fait suite au colloque tenu les 13 et 14 septembre 2013 à Berbiguières et interroge le rôle joué par les routiers et les mercenaires durant la guerre de Cent ans. Il cherche à combler un " vide historiographique ": les études menées au tournant de la fin du XIX et du début du $\mathrm{XX}^{\mathrm{e}}$ siècle, ont été ensuite abandonnées côté français alors qu'elles ont été poursuivies côté britannique. D'autres pistes sont aussi abordées et développées à travers les 16 contributions individuelles : com- 
ment définir ce groupe de combattants aux contours incertains? Comment rendre visible la diversité des parcours et des profils en croisant les approches des sciences humaines? Ce recueil constitue donc à la fois un point de départ et un appel à poursuivre les investigations engagées.

Les présentations des auteurs sont l'occasion de comparer les évolutions divergentes des deux grands protagonistes, la France et l'Angleterre, tout au long des XIV ${ }^{\mathrm{e}}$ et $\mathrm{XV}$ e siècles à travers le mercenariat. Jean-Philippe Genêt souligne fort à propos en introduction que les structures sociales et politiques des deux pays étaient quasisimilaires au début de la période avant de connaître des destins divergents à la veille des Temps modernes avec la création d'une armée de métier en France. Ce fait a eu des répercussions politiques sur la construction de l'État moderne, notamment pour ce qui a trait à la " domestication des élites " opérée à travers les carrières militaires.

La bibliographie très fournie, pas moins de 542 références, est un autre atout de cette publication. Le lecteur non-averti pourra toutefois regretter le classement adopté, par ordre alphabétique, qui complique le repérage des références incontournables. Un index aurait aussi été le bienvenu pour parcourir plus aisément et recouper les notices insérées dans les contributions, en particulier celles à caractère prosopographique. Ceci n'altère néanmoins en rien la qualité du recueil, agrémenté de cartes de bonne, voire de très bonne facture (p. 24, 35, 128 et 167), ainsi que de planches en couleurs soulignant l'intérêt des documents choisis par les auteurs (p. 60-61, 292-294 et 299).

L'ensemble est constitué de deux sortes de contributions : des présentations thématiques de dimension souvent générale - Guilhem Pépin, Justine FirnhaberBaker, Nicolas Savy, Pierre Prétou, Michael Jones, Germain Butaud, Françoise Lainé, Christophe Masson, Bertrand Schnerb, Werner Paravicini et Anne Curry et d'autres axées sur des personnages révélateurs des enjeux (Armand Jamme, Philippe Contamine, Loïc Cazaux, Valérie Toureille et Kelly de Vries). Nombre de communications se terminent par la présentation en annexes de pièces justificatives, d'un intérêt tout particulier pour les chercheurs, car pour la plupart inédites et présentées avec un appareil critique développé.

Guilhem Pépin aborde la question des routiers gascons, basques agenais et périgourdins du parti anglais (p. 23-56). Il met en évidence les motivations, les origines, les rayons d'action ou encore la perception qu'avaient les populations locales de ces routiers. Il insiste en particulier sur la difficulté qu'il y a à saisir ces hommes aux comportements mouvants selon les circonstances et les opportunités. Armand Jamme présente Bernard de La Sale, tour à tour au service de l'Angleterre et du pape (p. 57-99). Il examine les éléments qui permettent de transformer un chef de guerre aux origines incertaines en un personnage reconnu, intégré par les pouvoirs dans leurs calculs politiques et leurs institutions de gouvernement du fait même de ses actions et de ses succès militaires lors de ses coups de main spectaculaires (prise de Figeac en août 1371). Nicolas Savy s'intéresse aux procédés tactiques des compagnies anglo-gasconnes entre Garonne et Loire dans les années 1350-1400 (p. 115131) pour expliquer comment des compagnies, pourtant inférieures en nombre et vivant sur le pays ennemi, ont dominé militairement durant plusieurs décennies en s'adaptant constamment et en préparant méticuleusement leurs attaques. Cette contribution, très riche d'exemples, décortique ces mécanismes, en particulier en matière de renseignement.

Justine Finrhaber-Baker revient sur les impacts des actions des soldats sur les campagnes et sur la révolte contre les nobles du nord de la France lors de la Jacquerie de 1358 (p. 101-114). Elle insiste en particulier sur les craintes suscitées par les échos des violences parmi les paysans, qui ont convaincu certains de s'engager dans la révolte même si les territoires ne faisaient pas tous l'objet de 
déprédations militaires directes. Pierre Prétou axe son propos sur les réactions communautaires en Gascogne (p. 133-145), soulignant le fait que les paysans, loin d'être victimes, étaient aussi acteurs des violences et organisaient leur défense par des cris d'appel manifestant leur solidarité.

Philippe Contamine aborde les aspects politiques (p. 147-164). Jehan de Blaisy, auquel il consacre son étude, contribue par son action envers les compagnies des forteresses d'Auvergne à faire émerger une " certaine conception de l'État " (Françoise Autrand) où les compagnies n'ont plus leur place, car elles constituent autant de menaces et de freins à l'affirmation de l'État. Dans le même registre, en partant des archives et des chroniques bourguignonnes, Loïc Cazaux cherche à caractériser l'action d'Antoine de Chabannes, chef notoire des Écorcheurs (p. 165177). L'Écorcherie a en effet de multiples résonances politiques et diplomatiques après les négociations du traité d'Arras qui conduit au rapprochement entre les rois de France et les ducs de Bourgogne. A. de Chabannes, issu de la noblesse moyenne, constitue un point de départ pour appréhender et explorer ces enjeux dans les années décisives 1430-1440. Valérie Toureille poursuite la démarche en se focalisant sur Robert de Sarrebrück, seigneur lorrain encore mal connu aujourd'hui (p. 179187). Ce routier au service de Charles VII dirige des opérations de pillage sur les frontières orientales du royaume, dans des régions disputées, où se mêlent intérêts personnels et service de la monarchie française sans qu'il soit toujours possible de bien faire la part de l'un et de l'autre. Ce rôle pour le moins ambigu se retrouve dans la présentation de Perrinet Gressart par Kelly de Vries (p. 227-237). Ce chef de guerre bourguignon et anglais se rallie au roi de France dans les années 1440, ce qui lui permet de conserver son statut de défenseur de la garnison de La Charité-sur-Loire.

Michael Jones (p. 189-205), Germain Butaud et Françoise Lainé (p. 239-256) étudient les trajectoires de quelques mercenaires au service du duc de Bar en 1372-1373, durant la guerre civile de Provence (1383-1388) ou entre 1338 et 1441 en Gascogne, pour mieux cerner le milieu militaire actif dans ces affrontements. Les dernières contributions de Christophe Masson (p. 257-266), de Bertrand Schnerb (p. 267-276), de Werner Paravicini (p. 277-301) et d'Anne Curry (p. 303-316) n'évoquent pas autre chose en s'intéressant en particulier à l'origine et à l'importance de ces hommes dans les combats engagés tout au long $\mathrm{du}_{\mathrm{Xv}}^{\mathrm{e}}$ siècle, où ils se révèlent être à la fois des atouts indispensables pour faire nombre et pour pouvoir l'emporter, mais aussi des éléments perturbateurs qui contribuent paradoxalement à renforcer les outils et les structures à la disposition de l'État pour imposer son autorité. Werner Paravicini s'intéresse aussi à un espace périphérique par rapport à la guerre de Cent ans, avec le " voyage de Prusse ", qui lui permet de revenir sur la question du débordement des conflits en dehors du cadre strict de la guerre de Cent ans et de l'espace français.

$\mathrm{Au}$ total, ce livre fournit de nombreux exemples, souvent riches et avec des approches inédites, pour initier de nouveaux travaux.

Brice RABOT

CHOLLet, Mathilde, Etre et savoir. Une ambition de femme au siècle des Lumières, Rennes, PUR, coll. « Histoire », 2016, 302 p.

Beau titre pour un beau livre! À partir d'une recherche originale dans les archives publiques (minutier central des Archives nationales, archives départementales de la Sarthe et celles du Loir-et-Cher) Mathilde Chollet nous donne l'étonnant portrait d'Henriette-Françoise Edme (de Marans), née en 1719 dans une famille de 
bourgeois devenus gentilshommes de Cormenon (Perche, act. Loir-et-Cher) mariée en 1755 à Louis-Joseph de Vanssay, un membre d'une famille d'ancienne noblesse, et qui meurt, sans enfants, en 1784. Un arbre généalogique, hélas très difficile à lire en raison de la police de caractères choisie, expose sa parentèle car il faut savoir qu'Henriette, vit au château des Rouaudières avec sa nièce Marie-Pascale, dite Pascalitte (1756-1835).

L'ouvrage est divisé en trois parties (Comment les savoirs viennent aux femmes?; Journal et identité. De la quête personnelle à la revendication sociale ; Les choix d'une ambitieuse? L'admirable normalité de madame de Marans) et sept chapitres (Instruire les filles des Rouaudières : deux modèles, deux enjeux différents ; La sociabilité au château, apprentissage des plaisirs et plaisirs de l'apprentissage; Les influences spirituelles de madame de Marans, entre morale et philosophie; La médecine de l'âme. La recherche d'un système conciliant le moi et la foi; Madame de Marans et l'identité nobiliaire. Un discours féminin sur l'excellence; S'approprier et entretenir des pratiques culturelles interdites?; Une réflexion sur la place de la femme dans la société des Lumières). Les intitulés des chapitres donnent le ton du livre qui est une plongée sensible et documentée dans l'univers de cette femme qui cherchera, sa vie durant, à s'instruire et à comprendre le monde qui l'entoure.

Pour mener cette enquête, l'auteur a disposé de sources inédites en trois volumes, qui forment quasiment une autobiographie : Mémorial à mon usage particulier, Réflexions journalières et Confidences générales et particulières. À cela il faut ajouter nombre d'écritures ordinaires, ces papiers de famille - notamment la correspondance active de $\mathrm{M}^{\mathrm{me}}$ de Marans avec sa nièce Pascalitte - qui établissent le système d'écriture original d'une femme de lettres n'hésitant pas non plus à franchir les limites de la fiction en publiant en $1758 \mathrm{chez}$ le fameux libraire parisien Siméon-Prosper Hardy Les pensées errantes avec quelques lettres d'un Indien, roman épistolaire attribué encore aujourd'hui, par la notice d'autorité de la BnF à madame de Bénouville. La démonstration de Mathilde Chollet qui propose une autre attribution, à madame de Marans cette fois-ci, est convaincante. Après avoir fait une biographie synthétique de son héroïne, l'auteur fait le point sur l'historiographie du sujet, entre micro-histoire, histoire des femmes, histoire des pratiques d'écriture et approche socioculturelle des élites rurales de l'Ouest à l'époque moderne. Ces quelques pages, pour être brèves, sont néanmoins très intéressantes car elles nous montrent la richesse et la diversité des recherches menées actuellement sur ces sujets. Outre la découverte de la personnalité de madame de Marans, l'acquis principal de ce livre réside en effet dans l'étude de la question de l'éducation des femmes des femmes de la petite noblesse provinciale au XvIII ${ }^{e}$ siècle à l'époque moderne, encore bien mal connue. Le premier chapitre décrit par le menu la vie de madame de Marans et son souci constant de mener à bien l'instruction de sa nièce. Plusieurs membres de la petite noblesse créent et entretiennent des fondations religieuses destinées, entre autres, à instruire les filles. On trouve aussi à Vendôme des institutions comme le collège des Ursulines ou celui du Calvaire, où s'enseignent, outre la lecture, l'écriture et les travaux d'aiguille mais aussi l'arithmétique. Il y a davantage d'institutions à Blois et les cousines de Pascalitte, venues de Saint-Domingue, sont envoyées à Nantes ou à Bordeaux, métropoles plus importantes qui comptent davantage d'institutions pour un enseignement plus approfondi et plus diversifié. L'examen des catalogues des bibliothèques privées de ces familles montre également la présence de nombreux livres touchant à la religion, mais aussi à la littérature et à l'art épistolaire, avec des exemples tirés de lettres de madame de Sévigné. Ici encore il se vérifie que la réputation de la marquise a été quasiment synchrone avec l'écriture de ses lettres, dont on sait qu'elles pouvaient être recopiées, puis lues publiquement à peine écrites! 
Les chapitres suivants décrivent la vie quotidienne au château, les loisirs et les pratiques de sociabilité qui assument également un rôle culturel et une fonction éducative. Ici, Mathilde Chollet étudie les lettres de madame de Marans, le contenu de cette correspondance mais aussi les "réseaux du cœur ", réseaux d'amitié, de voisinage encore plus que de famille. Puis elle étend son étude aux conversations, aux jeux de société, aux travaux des champs, aux considérations sur les vêtements etc. Dans la famille Edme, l'éducation des filles est donc valorisée car c'est un signe de distinction sociale. Avec l'histoire, la morale est le sujet de prédilection de madame de Marans qui se soucie aussi de philosophie et de son édification spirituelle. Elle lit et commente les auteurs latins (on verra une passion semblable quelque trente ans plus tard chez Rosalie Jullien, issue de la bourgeoisie aisée des environs de Paris). De nombreux exemples dans ce livre montrent comment se construit une personnalité à l'âge des Lumières, à partir de l'héritage de l'Antiquité et des exempla de l'âge classique, le XvII ${ }^{e}$ siècle. Épistolière elle-même, aimant réfléchir sur la société, elle se revendique de La Bruyère car son " Journal " n'est ni un repli sur soi ni une simple introspection mais une base pour un programme d'amélioration morale afin de mettre en miroir ses pensées et sa conduite personnelles face au monde extérieur. Un chapitre entier, joliment intitulé "La médecine de l'âme " est consacré à la recherche par madame de Marans d'un système conciliant le " Moi " et la Foi. On y voit une grande liberté vis-à-vis de certaines croyances de l'Église catholique.

Issue par mariage d'une moyenne noblesse de l'Ouest fraîchement anoblie, madame de Marans, née de la bourgeoisie parisienne, fréquente la bourgeoisie d'office de Mondoubleau. Femme, elle fréquente les autres femmes. Mathilde Chollet examine les voies de la construction d'une identité nobiliaire toute récente et montre qu'elle dédaigne les catégories sociales inférieures tout en enviant les catégories sociales supérieures à elle. En cela, son héroïne est parfaitement conforme au monde qui l'entoure car, si l'anobli partage les codes du second ordre, il doit observer une certaine réserve vis-à-vis de la noblesse de haut rang. Lorsqu'elle évoque le mariage de son frère avec une personne d'extraction sociale inférieure, elle montre comment se fait le changement progressif des valeurs, le passage entre ce qui est de la naissance et ce qui ressort du mérite. Il y a là une fusion entre les valeurs aristocratiques anciennes et les valeurs dites bourgeoises. Mathilde Chollet conclut ces pages ainsi : «Dans les années 1780, c'est l'évolution de cette conception de la société qui contribue, en partie, au besoin revendiqué par l'opinion publique de réviser la hiérarchie sociale ".

Madame de Marans se comporte aussi en " citoyenne éclairée ", aspirant à une monarchie tempérée qui tire de l'Histoire, mais aussi des faits d'actualité, son idéal politique. Elle s'insurge contre Cicéron, examine dans le détail Montesquieu, donne son avis sur tous les auteurs qu'elle semble lire avec passion, commente l'actualité religieuse et en particulier la question du jansénisme, plus dans sa dimension politique que religieuse d'ailleurs car la question de la foi ne semble guère l'interpeller! À travers ses lectures, et les explications qu'en donne l'auteure, on voit que l'objectif de madame de Marans n'est rien moins que d'atteindre la perfection et au bonheur. La morale fait partie de la culture mondaine, au XVII ${ }^{\mathrm{e}}$ et au XVIII ${ }^{\mathrm{e}}$ siècles et ses références sont les auteurs de l'Antiquité, Horace, Térence, Cicéron, les Pères de l'Église, en particulier saint Augustin mais aussi les moralistes du Grand siècle, La Bruyère ou La Rochefoucauld, d'où ses discours sur la raison et sur les passions. Enfin, membre de la petite noblesse rurale, elle se doit de se distinguer à la fois du peuple, des roturiers, des bourgeois aisés des petites villes et de la paysannerie, qu'elle côtoie puisqu'elle s'occupe de la gestion du domaine. Femme aux idées politiques et sociales modérées, elle n'en est pas moins une ambitieuse dans le domaine intellectuel. Est-elle féministe pour autant? Échappant aux embûches 
théoriques d'un sujet trop souvent malmené, Mathilde Chollet annonce la couleur avec le titre, accompagné d'un point d'interrogation, de la troisième partie de son livre « Les choix d'une ambitieuse? L'admirable normalité de madame de Marans ". Se voulant avant tout une femme d'esprit et être considérée comme telle, madame de Marans est réaliste et mesure les contraintes culturelles, sociales et psychologiques pesant sur les femmes. Son féminisme, si féminisme il peut exister, serait un " pessimisme égoïste " (p. 244-248) tant elle n'imagine pas possible de changer les inégalités sociales qu'elle observe...

Pour conclure, les documents du for privé de madame de Marans judicieusement retrouvés, contextualisés et analysés par Mathilde Chollet apportent beaucoup à la connaissance de ces femmes d'une petite aristocratie provinciale beaucoup moins connue que les salonnières parisiennes. Enfin, l'auteur nous livre des réponses inédites, enrichissantes et nuancées dans le domaine de l'histoire intellectuelle ou de l'histoire des idées, notamment sur la diffusion et la réception des Lumières.

Annie DUPRAT

BOUGEARD Christian, PRIGENT François (dir.), La Bretagne en portrait(s) de groupe. Les enjeux de la méthode prosopographique (Bretagne, XVIII ${ }^{e}-X X^{e}$ siècle), Rennes, PUR, coll. « Histoire ", 2016, 339 p.

À l'heure actuelle, le traitement quantitatif de larges enquêtes ainsi que le développement des dictionnaires et des bases de données en ligne témoignent de l'intérêt croissant pour la prosopographie, quelle que soit la période historique envisagée. C'est dans cet allant que s'inscrit l'ouvrage dirigé par Christian Bougeard et François Prigent, résultat d'une journée d'étude et d'un colloque organisés à l'université de Bretagne occidentale (Brest) en 2013 et 2014, avec le soutien de l'association Maitron Bretagne et du Centre de recherche bretonne et celtique (CRBC). L'ouvrage se divise en trois parties : les deux premières sont consacrées à l'univers politique et la dernière aux élites religieuses, militaires et culturelles. Afin de donner du sens à un travail collectif qui rassemble les portraits de groupes, les directeurs ont choisi la Bretagne historique, à cinq départements : au-delà de sa forte identité et de sa cohérence sur le " plan des évolutions géopolitiques et des structures sociales " (p. 9), elle dispose de nombreux travaux de qualité sur les sujets qui les préoccupent. D'emblée, la volonté affichée est de faire intervenir les jeux d'échelles et de comparer la région à d'autres espaces.

L'ouvrage rassemble une vingtaine de communications écrites par des universitaires, des docteurs et des doctorants mais aussi des étudiants de master 2, des archivistes ou encore des chercheurs en poste dans l'enseignement secondaire. Cette diversité a pour conséquence des niveaux de réflexion et d'écriture très différents selon les articles. Certains s'inscrivent dans des recherches achevées et effectuent un retour d'expérience sur les procédés utilisés pour mener l'enquête (pour ne citer qu'eux, François Prigent ou Samuel Gicquel), lorsque d'autres font état de questionnements méthodologiques inhérents à toute réflexion naissante ou adolescente (à titre d'exemple, le texte de Blandine Lefierdebras et Lydie Porée). Il serait vain de dresser ici un résumé de chacun des articles, tant les sujets traités sont divers. Nous préférons nous concentrer sur ceux qui ont plus particulièrement retenu notre attention par leur questionnement, leur démarche ou leurs résultats.

Deux textes introductifs sur la méthodologie (François Prigent) et l'historiographie (Éric Anceau) de la prosopographie la définissent tour à tour comme un outil, 
une méthode voire une façon d'écrire l'histoire. Les deux auteurs mettent aussi en garde sur les limites, voire les illusions, qu'elle peut susciter. Mais, entre apports qualitatifs et analyses quantitatives, entre trajectoires individuelles et portraits collectifs, la démarche prosopographique peut être d'une grande richesse et, notamment, apporter à la connaissance et la compréhension des carrières et des réseaux.

La première partie de l'ouvrage analyse les élites politiques, entendues ici comme les notables et les élus bretons. Des dirigeants du PCF de 1944 à 1968 (Paul Boulland) aux parlementaires gaullistes (Christian Bougeard et Olivier Herbinet) en passant par les parlementaires socialistes (François Prigent) ou les militants écologistes (Martin Siloret) c'est un large spectre des cultures politiques qui est étudié, avec une nette prédominance pour le $\mathrm{xx}^{e}$ siècle. L'approche prosopographique permet à Christian Bougeard de constater qu'entre 1951 et 1981, les députés gaullistes sont majoritairement issus des élites sociales et sont dotés d'un " fort capital culturel, social et professionnel " (p. 88). Dans l'évolution des carrières, ce sont leurs compétences professionnelles, leur notabilité élective et leur enracinement local qui priment. Les deux seuls textes dont l'objet d'étude se situe plus en amont sont celui de Bruno Baron, qui se penche sur la trajectoire d'une famille d'édiles brestois de la fin du XVII ${ }^{\mathrm{e}}$ au début du XIX ${ }^{e}$ siècle, et celui d'Anne de Mathan et Philippe Jarnoux. S'inscrivant dans un projet ANR en cours (" Acteurs et actions politiques en Révolution "), dont l'objectif est d'élaborer un dictionnaire des 749 conventionnels français, ils travaillent sur les profils des députés de la Convention élus dans les Côtes-du-Nord, le Finistère et le Morbihan de 1792 à 1795. Les deux historiens souhaitent mieux connaître les milieux d'origine de ces députés et suivre les évolutions de leurs positionnements politiques et de leurs carrières. Dès lors, il s'agit d'étudier un ensemble d'élus, à l'interface des influences locales et de la représentation nationale, qui a marqué l'histoire politique de manière durable ainsi que la nature des fonctionnements parlementaires (p. 75-76).

Dans un second temps, l'ouvrage effectue une plongée dans l'univers des militants. Là encore, si un article sur les fouriéristes finistériens s'ancre dans le XIX ${ }^{\mathrm{e}}$ siècle (Jean-Yves Guengant), c'est bien le siècle suivant qui est au centre des attentions, avec l'examen des trajectoires collectives des fusillés en Bretagne durant l'Occupation (Alain et François Prigent), des militants syndicaux - ceux de la CGT, de la CFTC et du SNI dans les Côtes-du-Nord de 1935 à 1965 (Alain Prigent), et du SEP-CFDT dans le Morbihan de 1957 à 1985 (Daniel Chérel et François Prigent) - ou encore des féministes rennais durant la deuxième vague du féminisme, des années 1960 aux années 1980 (Blandine Lefierdebras et Lydie Porée). Alain Le Moigne, quant à lui, étend sa réflexion sur le mouvement anarchiste au sein du monde ouvrier de l'arsenal brestois des années 1890 aux années 1930. Son article débute par la présentation et la critique de son corpus de base : qu'il s'agisse des notices de suspects établies par la police, celles des individus considérés comme dangereux pour la Nation ou encore le carnet B du département, toutes ces sources doivent impérativement être confrontées à d'autres tant elles surévaluent la menace de certains personnages durant les périodes de tensions et ne tiennent pas compte " de la réalité et de la diversité des sensibilités idéologiques " (p. 166). Au fil des pages, le contraste apparaît énorme entre l'importance du sentiment subversif provoqué par l'anarchisme brestois et la réalité : les travailleurs anarchistes n'ont jamais été qu'une poignée (entre 20 et 120) pour l'ensemble du Finistère durant la période analysée. Plus que l'anarchisme, ce sont les sentiments antimilitaristes et pacifistes qui priment au sein du prolétariat brestois.

Le troisième et dernier acte questionne les élites culturelles, militaires et religieuses bretonnes. Cette fois, le large $\mathrm{XIX}^{\mathrm{e}}$ siècle occupe une place non négligeable. Tour à tour, ce sont les folkloristes (Laurent Le Gall), le $47^{\mathrm{e}}$ Régiment d'infante- 
rie pendant la Première Guerre mondiale (Erwan le Gall), les évêques bretons au $\mathrm{xx}^{\mathrm{e}}$ siècle (Frédéric Le Moigne) ou encore les responsables de la Jeunesse Étudiante Chrétienne durant les Trente Glorieuses (Bernard Giroux) qui sont étudiés. Les études de Samuel Gicquel et Manon Le Guennec sont à signaler. Le premier évoque son utilisation de la prosopographie lors de sa thèse de doctorat consacrée aux milliers de prêtres concordataires bretons. Ouvrant son texte sur les difficultés méthodologiques, parfois chronophages, auxquelles il a dû faire face pour relever le " défi de la masse " (p. 240), il met ensuite en évidence la singularité des carrières bretonnes et quelques destins extraordinaires, ces derniers étant toujours finement replacés dans le collectif. La seconde dessine les contours et appréhende le fonctionnement du monde des professeurs du lycée de Rennes de 1870 à 1914 (p. 292). Scrutant leurs milieux sociaux et professionnels d'origine, la place du lycée rennais dans leur carrière ou encore l'action de l'administration sur ces derniers, elle dégage un certain nombre de traits si ce n'est communs, du moins partagés. On apprend par exemple que les membres de ce corpus sont pour une large part issus des catégories populaires et ont de véritables stratégies afin de gravir le cursus honorum professoral, stratégies dans lesquelles le lycée de Rennes n'est bien souvent qu'une étape.

En conclusion, Gilles Richard rappelle les forces de l'ouvrage - notamment sa capacité à faire émerger des interrogations et des pistes nouvelles - tout en invitant les historiens à ne pas surévaluer les chiffres et les portraits-types. Sousgroupes, exceptions et contradictions sont aussi à traquer. Tout en abondant dans ce sens, il nous appartient d'émettre une réserve en ce qui concerne la cohérence de l'ensemble. En refermant l'ouvrage, on ne sait trop ce qu'est son véritable objet : prosopographie ou histoire de la Bretagne. Tous les auteurs n'utilisent pas la même définition de la prosopographie, ce qui rend leurs enquêtes difficiles à penser ensemble. Force est même de constater que certains ne font pas état de recherches prosopographiques à proprement parler, si du moins l'on fait sienne la définition de François Dosse, pour lequel la prosopographie "se donne pour objet de restituer les caractéristiques d'un groupe en démultipliant les informations sur tous ses membres. Ne s'attardant pas sur la singularité de chacun de ses éléments, [elle] cherche à fonder statistiquement les archétypes " (dans Delacroix, Christian et al. [dir.], Historiographies, I. Concepts et débats, Paris, 2010, p. 79-85).

Si cet ouvrage ne livre une réflexion unifiée ni sur l'histoire de la Bretagne ni sur la méthode prosopographique, toujours est-il que nombre des études de cas qu'il contient apportent ponctuellement à la connaissance de l'une et à la compréhension de l'autre.

Fabien LosTEC

CARnEY, Sébastien, Breiz Atao! Mordrel, Delaporte, Lainé, Fouéré : une mystique nationale (1901-1948), Rennes, PUR, coll. « Histoire », 2015, 608 p.

C'est une relecture particulièrement intéressante du nationalisme breton que propose Sébastien Carney dans cet ouvrage, solide pavé composé autour des parcours biographiques croisés de quatre de ses figures emblématiques. Au sein d'une bibliographie déjà très riche sur le sujet, il réussit, en effet, à apporter de nouveaux éclairages dont on peut déjà affirmer qu'ils vont s'avérer incontournables pour toute tentative de compréhension de ce mouvement dans la première moitié du Xx $\mathrm{x}^{\mathrm{e}}$ siècle.

$\mathrm{Au}$ cœur de cette réussite, la rigueur d'une démarche biographique qui cherche, à travers ces parcours de vie, à éclairer l'histoire du mouvement breton en explo- 
rant les tréfonds de certains engagements individuels ainsi que les concours de circonstance qui les déterminent. Sébastien Carney fait alors de ces hommes les représentants d'une génération spécifique marquée par l'événement matriciel de la Grande Guerre. Trop jeunes pour l'avoir faite - Olivier Mordrel(le) est né en 1901, Raymond Delaporte en 1907, Célestin Lainé en 1908 et Yann Fouéré en 1910 - ces jeunes gens l'ont pourtant vécue, enfant ou adolescent, avec intensité tout comme ils ont ressenti durement, par la suite, le fait de ne pas y avoir participé, portant comme un fardeau l'héroïsme de leurs aînés. Cette frustration de ne pas avoir connu la guerre engendre chez eux un " complexe de Mars " qui les pousse alors à reporter leur ardeur sur la cause nationale bretonne. Si cette approche psychologique, comme clé de lecture, peut paraître parfois un peu systématique, notamment dans l'insistance qu'elle porte à leurs rapports avec père et mère, elle n'en permet pas moins de dépasser une approche purement idéologique de leur engagement nationaliste ouvrant ainsi de stimulantes perspectives.

Dans la première partie qu'il intitule "rêves ", Sébastien Carney étudie comment se construit l'identité bretonne de ces différents personnages pour lesquels elle n'a rien d'évident, ne serait-ce que parce qu'aucun d'entre eux n'a la langue bretonne comme langue maternelle. Loin donc d'une approche essentialiste de cette identité, si souvent agitée par les acteurs de l'emsav, hier comme aujourd'hui, il montre comment celle-ci prend corps chez ces jeunes gens, au lendemain de la Grande Guerre, dans un rapport conflictuel à des pères " devenus monuments écrasants " et dans une espérance de renouvellement des horizons politiques et intellectuels. Sorte de catharsis, la cause bretonne leur permet d'exprimer à la fois le rejet du monde matériel et sans âme que symbolise, pour eux, la France - la haine de la France pouvant être lue comme le rejet du père - et des aspirations spirituelles confuses où l'apologie de l'âme celtique se mêle à des considérations raciales appelées à devenir racistes. La Bretagne comme refuge donc, pour ces jeunes hommes qui trouvent ici le combat et les perspectives exaltantes dont ils se sentaient jusque-là frustrés. Sébastien Carney souligne cependant que si le nationalisme breton ouvre une voie, celle-ci est empruntée avec de nombreux tâtonnements et qu'elle n'opère pas, pour tous, de la même façon. On perçoit clairement, grâce à l'évocation de ces différentes trajectoires, notamment celle de Célestin Lainé, la complexité, les hésitations et les balbutiements de ces engagements nationalistes où les tensions psychologiques et les rêves romantiques agissent sans doute bien plus fortement que toute considération idéologique clairement établie. Ce qui compte est sans doute moins le but que la lutte elle-même. Il n'empêche que tous se retrouvent autour de Breiz Atao, organe emblématique du nationalisme breton, autour desquels vont s'organiser, tout au long de la période étudiée par l'auteur, leurs rapprochements, leurs rivalités et leurs divergences, parfois fortes, notamment sur la place que doit prendre le catholicisme dans le " relèvement " de la Bretagne. C'est du reste avec Breiz Atao que ces différentes personnalités informent sur le petit monde du nationalisme breton qui s'agite alors autant sur le plan intellectuel, par la création de multiples revues, que politiques par la création de plusieurs formes partisanes dont le Parti national breton (PNB) à la fin de l'année 1931. C'est ce moment que Sébastien Carney choisit pour replacer leur action dans le fourmillement des "relèves " et des " non conformistes " qui marque le paysage intellectuel, plus que politique, de la France et de l'Europe dans le projet d'une révolution conservatrice pensée comme une troisième voie. En invitant ainsi à dépasser le strict environnement de la Bretagne et en replaçant ces engagements nationalistes dans le cadre des "relèves ", l'auteur propose ici des approches particulièrement novatrices qui font tout l'intérêt et l'originalité de son travail. 
Le passage du " rêve " aux réalisations qui se produit alors définit des "élévations " qui sont l'objet de la deuxième partie de l'ouvrage. Désormais, œuvrer pour "l'émancipation de la Bretagne "s'effectue de manière résolue autant dans une dimension politique radicale, symbolisée par l'attentat contre le monument de la place de la Mairie de Rennes, que dans une dimension intellectuelle où s'affirment de manière croissante les tropismes celtiques et nordistes. C'est dans ce contexte que certains tournent leurs regards vers l'Allemagne nazie dans la recherche et l'exaltation d'une mystique nationale. Si leurs engagements prennent parfois des formes plus pragmatiques, à l'exemple d'un Yann Fouéré et de son action en faveur de la promotion de la langue bretonne, la radicalisation et la racialisation de leurs convictions nationalistes, l'aspiration à la violence que développe, par exemple, Célestin Lainé, ouvrent la voie aux abjections qui vont caractériser leurs actes durant la Seconde Guerre mondiale.

Cette dernière période est bien celle des " vertiges " qu'évoque dans sa troisième partie Sébastien Carney. Sans doute ici l'ouvrage est-il moins novateur tant l'attitude de l'emsav durant cette période a été parcourue avec intensité par l'historiographie. L'approche biographique ne renouvelle pas véritablement - mais était-ce possible? - ce que l'on sait des actes de ces militants nationalistes pris dans l'ivresse d'une possible consécration de leur cause et de leur ego. Vertige donc qui les saisit devant la victoire des armées nazies et la défaite de la France abhorrée, vertiges entretenus par l'écoute attentive que leur offre, un temps, l'occupant et qui les conduit à la déclaration tonitruante d'un Conseil national de Bretagne, ne représentant qu'euxmêmes, à Pontivy en juillet 1940. Si l'évolution de l'attitude des autorités nazies à leur égard est rapidement source de désenchantement, ils n'en cherchent pas moins, par tous les moyens, à se placer en position de pouvoir et d'influence sur fond de rivalités, parfois mises en scène. Il en est ainsi de la rivalité surjouée entre L'Heure bretonne dirigée un temps par Mordrel et La Bretagne que Fouéré prend en main. Mais le revirement de l'occupant plus désireux de ne pas froisser Vichy que de favoriser les nationalistes bretons oblige ces derniers à un repli sur soi porteur, là encore, de vertiges. Vertiges idéologiques d'une puissance rêvée où haine de soi et haine des autres se mêlent au mysticisme et à la violence pour déboucher, à la fin de la guerre, sur les compromissions les plus odieuses avec les nazis à l'instar d'un Lainé et de son unité Perrot. Vertiges crépusculaires que seul l'instinct de survie transcende dans la fuite honteuse puis la dissimulation avant que d'entreprendre, avec d'autres, une longue entreprise de réhabilitation non dénuée, il faut le regretter, d'une certaine réussite.

Solidement établi sur des sources nombreuses et multiples, étayé par une solide bibliographie, l'ouvrage de Sébastien Carney renouvelle donc en profondeur et avec bonheur l'historiographie du nationalisme breton.

David BENSOUSSAN

Noppen, Luc, Coomans, Thomas, Drouin, Martin (dir.), Des couvents en héritage. Religious houses: a legacy, Québec, Presses de l'Université du Québec, 2015, $501 \mathrm{p}$.

Depuis les années 1990, les historiens québécois forment l'avant-garde de la réflexion sur le devenir du patrimoine de l'Église catholique. Cela s'explique par la brutalité et la force du retournement religieux au Québec, ce qui a posé précocement la question de la réaffectation de lieux de culte. S'en sont saisis en particulier 
Luc Noppen et Lucie K. Morisset, deux historiens de l'architecture, professeurs à l'université de Québec et organisateurs des colloques " La conservation des églises dans les villes-centres " en 1997 et "Quel avenir pour quelles églises? " en 2005. Le présent ouvrage est la publication des actes d'un troisième colloque international, qui s'est tenu en octobre 2009 sur le sort des couvents au gré des sécularisations et des changements de propriétaires.

On peut s'étonner que l'édition des actes n'intervienne que six ans plus tard, ce qui est d'autant plus regrettable que plusieurs articles font référence à l'actualité de l'époque et s'interrogent sur la pertinence de projets alors débattus. Ce délai se comprend toutefois en feuilletant le produit fini, d'une qualité remarquable. Tous les articles sont illustrés d'une riche iconographie en couleurs, qui immerge le lecteur dans les études de cas développées, y compris lorsqu'il s'agit d'espaces dont il est peu familier. Un simple survol des illustrations permet d'aller du monastère SainteCatherine du Sinaï, fondé au $\mathrm{Vl}^{\mathrm{e}}$ siècle, aux énormes ensembles conventuels québécois récemment réhabilités, en passant par les abbayes médiévales européennes et offre ainsi un condensé de l'histoire du monachisme catholique.

L'ouvrage s'articule en deux parties inégales. La première, la plus courte, ne rassemble que deux contributions, il est vrai fort volumineuses (174 pages à elles deux). Plus qu'une recherche novatrice, le texte de Thomas Cooman est une longue introduction visant à expliquer comment l'architecture monastique a évolué au fil du temps et varié en fonction des règles de vie. Elle est complétée par un article cosigné par Luc Noppen et Lucie K. Morisset qui font une typologie de la conversion des monastères québécois et présentent les enjeux des opérations immobilières. L'article développe en particulier des réflexions fort intéressantes sur le sort des espaces verts enserrant les couvents urbains, qui sont dans certains cas davantage au cœur des luttes patrimoniales et financières que les bâtiments eux-mêmes.

La seconde partie, divisée en quatre sous-parties, suit un plan un peu artificiel. Il faut surtout la prendre comme un ensemble d'études (14 au total, tantôt en français, tantôt en anglais) portant sur des espaces et des périodes différentes, qui montrent la multiplicité des situations. Trois cas très divers sont présentés dans la première sous-partie, intitulée " Les expériences : motifs de l'affection patrimoniale ». Au Mexique, analysé par Ana Lozano Santin, la question du sort des couvents s'est posée dès la politique de nationalisation initiée en 1859, tandis qu'au Québec (Martin Drouin), leur requalification et la prise de conscience de leur valeur patrimoniale intervient surtout dans les années 1970. Pour la Russie, dont l'originalité est soulignée par Fabien Bellat, la question de la réoccupation des couvents se pose davantage que celle de leur désaffection depuis la chute du système communiste.

La juxtaposition de plusieurs communications dans une autre sous-partie consacrée aux " enjeux organisationnels " souligne la diversité des cadres de gouvernance. La Belgique flamande, décrite par Jan de Maeyer et Kristien Suenens, dispose par exemple d'instruments de conservation et de gestion tels que le KADOC, un centre de documentation et de recherche sur les rapports entre religion et société, lié à l'université de Louvain, qui est aussi un prestataire de service pour l'Église. Aux Pays-Bas, le musée d'art national et de culture a développé un livre de directives sur les manières de traiter les objets religieux (Marc de Beyer) et un groupe de travail a été constitué avec le soutien du gouvernement pour réfléchir au devenir du patrimoine religieux (Nico Nelissen). La situation française, marquée par la Révolution, est présentée à travers un prisme juridique par Anne Fornerod, qui développe plus précisément les exemples de Conques, Cadouin et du Mont-SaintMichel, tandis que Marie Lessard souligne l'importance du système urbanistique québécois dans la conservation du patrimoine conventuel en s'appuyant sur deux exemples montréalais. 
Parmi les contributions susmentionnées, beaucoup sont des réflexions assez générales, dont l'objectif est avant tout de proposer des typologies didactiques et des synthèses d'une situation nationale. L'ouvrage contient aussi des travaux de recherches sur des objets plus restreints, étudiés plus précisément, dans une souspartie intitulée " le temps long du patrimoine : conversion et reconversion ». Thomas Cooman analyse le sort de l'abbaye cistercienne de Villers-en-Brabant, ruinée en 1796 et Jean-Michel Leniaud celui de l'ancienne abbaye royale de Royaumont, qui fut notamment transformée en centre textile, en noviciat et en espace d'expression musicale. Dans un article qui intéressera tout particulièrement les passionnés d'histoire de la Bretagne, Yvon Tranvouez, en fin connaisseur des lieux, revient sur le destin des ruines de l'ancienne abbaye de Landévennec et analyse la variation de leur valeur symbolique au fil du temps.

Une sous-partie disparate sur les " postures du religieux dans la société contemporaine " conclut l'ouvrage. Isabelle Jouveaux décrit les conséquences de la patrimonialisation des monastères sur le système monastique, Geoffrey Carr l'incapacité des politiques fédérales à gérer l'héritage architectural du système des pensionnats amérindiens et Pierre Lucier " l'esprit du lieu ", c'est-à-dire la personnalité de l'habitat, reflet de la spiritualité et de la règle de vie en vigueur.

Le lecteur trouvera donc dans cet ouvrage quantité d'informations intéressantes sur le sort des couvents dans la chrétienté, mais on peut regretter l'absence de conclusion générale, qui aurait mis en perspective ces débats et souligné la diversité des situations : l'Europe occidentale, qui se passionne pour les ruines séculaires et demeure globalement attachée à la culture monastique, n'est pas le Québec, où des dizaines de bâtiments conventuels sont passés sous propriété privée ces dernières années, aiguisant les appétits des promoteurs.

Samuel GICQUEL

Pichot, Daniel, Provost, Georges (dir.), Histoire de Redon. De l'abbaye à la ville, Rennes, PUR/Société archéologique et historique d'Ille-et-Vilaine, 2015, 431 p.

Ce très élégant ouvrage collectif issu des actes d'un colloque organisé à Redon en octobre 2013 témoigne, s'il en était besoin, du savoir-faire des Presses universitaires de Rennes. Sa publication en coédition avec la Société archéologique et historique d'Ille-et-Vilaine permet en outre d'insister à nouveau sur la place qu'occupent les sociétés savantes bretonnes en matière de diffusion d'une partie de la recherche historique auprès du grand public. Le soutien de la Ville de Redon, du Conseil régional de Bretagne, celui de l'Association pour la protection du patrimoine historique de Redon, d'ailleurs à l'origine du projet de colloque, illustrent aussi ces synergies bretonnes fécondes au service de la connaissance dans un réel esprit d'ouverture et de collaboration avec les universitaires. Daniel Pichot, professeur émérite d'histoire médiévale à l'université Rennes 2, et son collègue Georges Provost, maître de conférences en histoire moderne, ont rassemblé une équipe associant historiens et archéologues afin de proposer une histoire de Redon renouvelée et résolument ancrée dans les problématiques récentes de l'histoire des villes. Illustration de ce que l'on peut appeler une histoire localisée et non pas seulement locale, elle vient incontestablement combler un vide historiographique longtemps dissimulé par le célèbre cartulaire qui certes fournit des éclairages exceptionnels pour les premiers temps de l'abbaye et de la ville mais a monopolisé « l'essentiel de la bibliographie récente" (p. 10). 
Au long de vingt-trois études très documentées, c'est toute l'histoire de Redon, de ses origines les plus lointaines - avant même la naissance de sa célèbre abbaye à l'orée du XXI ${ }^{\mathrm{e}}$ siècle, qui défile. Cette histoire est largement ouverte au patrimoine, qu'il soit naturel avec l'évocation du cadre hydrographique (chap. 1 : Marie-Josée Penven, Dominique Marguerie, « Les marais de Redon : la construction d'un paysage au fil des métamorphoses d'un fleuve "), monumental avec celle de l'abbaye (chap. 10 : Christophe Amiot, "L'abbaye Saint-Sauveur de Redon " et chap. 13 : Erwann Le Franc, "L'œuvre architecturale des bénédictins mauristes à l'abbaye de Redon "), mobilier avec celle des aménagements intérieurs de l'abbaye (chap. 14 : Roger Blot, "Dans l'abbatiale, le temps du mobilier "), immatériel avec celle de la fête de la châtaigne (chap. 23 : Martine Cocaud, "Les temps de la châtaigne. Du fruit nourricier à la fête de la Bogue "). Le recours aux ressources les plus neuves de l'archéologie permet quant à lui de restituer la lente mise en place du paysage de la basse vallée de la Vilaine et de son occupation (texte de Marie-Josée Penven, Dominique Marguerie, déjà cité mais aussi chap. 2 : Maurice Gauthier, Gilles Leroux, Arnaud Desfonds, Philippe Guigon, "Occupation protohistorique et réseau routier antique en aval de Redon "). Plus largement, l'ouvrage fait la part belle à la période médiévale objet de huit contributions, rayonnement de l'abbaye et cartulaire obligent, et aux temps modernes, étudiés par sept auteurs. Affaire de délais et/ou d'angle mort bibliographique, l'époque contemporaine semble un peu en retrait. Plusieurs encarts thématiques complètent avec bonheur le propos : "Redon en 1543 ", "Redon, diocèse éphémère " et "Redon et la Grande Guerre " (Daniel Pichot), "Les Pèlerinages à Redon " (Daniel Pichot et Georges Provost), "Les abbés commendataires " (dont Richelieu) et "Les couvents de Redon " (Georges Provost), "L'exil de la Chambre des comptes à Redon " (Dominique Le Page).

Les apports d'un tel livre sont par essence multiples et il n'est évidemment pas envisageable d'être exhaustif. On se contentera ici, rompant la logique chronologique qui est la sienne, d'évoquer quelques lignes de force qui structurent nombre de contributions et sont aussi les fils conducteurs dégagés par les directeurs de l'ouvrage. Le site et la situation de la ville, sa position de limite et de contact notamment, sont envisagés à chaque époque comme un facteur clé de son histoire en matière économique mais aussi politique ou religieuse. Ainsi, servie par sa position sur la Vilaine, "dont on a peine à imaginer aujourd'hui l'importance comme voie commerciale entre le proche Atlantique, la côte sud et l'intérieur de la Bretagne " (p. 198), la ville acquiert le statut d'avant-port de Rennes à la veille de la Révolution. La question de l'environnement naturel particulier dans lequel s'épanouit Redon ne peut donc elle non plus être éludée. Marais et considérations hydrographiques sont évoqués à plusieurs reprises sans être réduits à un simple arrière-plan, un cadre dans lequel se déploierait l'histoire de la ville. Au Moyen Âge, l'environnement aquatique est ainsi tout sauf répulsif et la ville étend son influence sur la basse Vilaine, notamment sur des marais qui "n'apparaissent donc ni putrides ni inhospitaliers " (p. 141) (chap. 8, Daniel Pichot : "Redon et l'eau, XIVe-Xvl e siècle "). Plus tard, à la fin de l'Ancien Régime, on comprend bien qu'ils constituent non seulement un espace aménagé mais aussi un espace convoité, au sein duquel les questions de délimitation, de mise en valeur, de conflits d'usage sont essentielles (chap. 17 : Sklaerenn Scuiller, "Les marais de Redon au XVIII ${ }^{\mathrm{e}}$ siècle, un paysage de conflits ").

Le rôle central de l'abbaye tout au long de ces 1200 ans d'histoire est légitimement scruté, depuis sa fondation par Conwoïon au IX ${ }^{\mathrm{e}}$ siècle dans un contexte politico-religieux troublé (chap. 3 : Caroline Brett, « Redon, abbaye carolingienne ") jusqu'à sa seconde vie comme " haut-lieu de l'enseignement secondaire catholique dans l'Ouest au XIX ${ }^{\mathrm{e}}$ siècle " recrutant dans tout l'ouest français sous la direction des Eudistes (chap. 21 : Gilbert Nicolas, "Le collège Saint-Sauveur de Redon "). 
Dès l'origine et assez rapidement, en s'appuyant sur son importante richesse foncière, l'abbaye a réussi à établir une vaste seigneurie ecclésiastique ainsi qu'un large espace d'échanges correspondant au bassin de la Vilaine (chap. 4 : Noël-Yves Tonnerre, « La puissance d'une abbaye carolingienne. Dominer la terre, dominer les hommes "). Soucieuse de s'insérer dans son environnement proche - local ou breton - Saint-Sauveur s'efforce en même temps de s'arrimer au monde franc. L'étude de sa production hagiographique et historiographique montre ainsi que, si son rayonnement culturel demeure limité, Saint-Sauveur est en revanche " perméable aux évolutions littéraires et diplomatiques (p. 96) " franques (chap. 5 : Claire Garault, "L'abbaye de Redon, entre horizon local et ouverture culturelle, $\mathrm{IX}^{\mathrm{e}}$-XII ${ }^{\mathrm{e}}$ siècle "). Dans un autre domaine, pendant la période de la réforme "grégorienne ", sous l'influence ambiguë des ducs de la maison de Rennes, les abbés, tout en insistant sur l'ancienneté et le prestige de leur abbaye et en mettant en valeur, de manière orientée certes, l'héritage carolingien, ont très tôt saisi tout l'intérêt qu'ils pouvaient retirer d'un rapprochement avec Rome (chap. 6 : Florian Mazel, « L'abbaye de Redon aux Xle-XII ${ }^{e}$ siècles. Entre mémoire carolingienne et réforme "grégorienne" "). Vient ensuite, entre le XIII et le Xve siècle, le temps du déclassement sous le double effet du renforcement de la tutelle ducale, que ce soit en matière judiciaire ou fiscale, et de l'accroissement de l'influence pontificale, perceptible notamment lors de l'élection des abbés (chap. 9, Vincent Corriol : "Après le cartulaire. L'abbaye Saint-Sauveur de Redon à la fin du Moyen Âge "). Plus tard, à partir de 1628 et après deux tentatives de réforme, "l'abbaye rejoint la dernière grande réforme bénédictine d'Ancien Régime, la congrégation de Saint-Maur (p. 201)". Au cours des XVII et XVIII siècles, Saint-Sauveur se distingue par l'importance accordée aux travaux historiques - la préparation des matériaux de l'Histoire de Bretagne, par exemple -, à la querelle janséniste et gallicane, à la prédication (chap. 12 : Daniel-Odon Hurel, « Saint-Sauveur de Redon dans la congrégation de Saint-Maur ").

Un ensemble de contributions permet de réfléchir plus spécifiquement au passage " de l'abbaye à la ville " suggéré par le sous-titre du livre. Le statut de la ville est alors interrogé. Aux époques médiévale et moderne, il s'agit d'étudier la vie dans une ville abbatiale depuis le moment où un centre urbain structuré s'est progressivement développé autour de son abbaye et de ses reliques. Son profil de ville portuaire et marchande au vaste arrière-pays, dominée par un pouvoir seigneurial monastique d'abord fort puis concurrencé par la montée en puissance de la communauté de ville, fait-il de Redon une ville atypique ou, au contraire, demeure-t-elle ordinaire? La question est successivement abordée par Julien Bachelier (chap. 7 : " Redon du IX ${ }^{\mathrm{e}}$ au milieu du XIV ${ }^{\mathrm{e}}$ siècle. Du centre abbatial à la ville médiévale "), Philippe Hamon (chap. 11 : "Redon pendant la Ligue [1589-1598]. Profiter de la guerre civile? »), Georges Provost (chap. 15 : "Vivre dans une ville abbatiale. Redon aux XVII ${ }^{\mathrm{e}}$ et XVIII ${ }^{\mathrm{e}}$ siècles ") et Philippe Jarnoux (chap. 16 : "Redon au XVIII ${ }^{\mathrm{e}}$ siècle. Une ville banale? »). Adhérant à la Révolution politique mais refusant la révolution religieuse (que l'on se souvienne du refus unanime du serment à la constitution civile par le clergé du district en 1790), la ville sera souvent le théâtre d'engagements en demi-teinte entre 1789 et 1799 . Au cœur d'un pays blanc, l'action des patriotes de Redon se fait alors modérée afin de leur permettre de se maintenir et de rallier l'opinion publique (chap. 18 : Solenn Mabo, "La Révolution à Redon "). Après la Révolution, la donne change. Redon, en dépit de sa modestie, s'affirme comme une ville à part entière. Ainsi, dans le champ économique, à la charnière des $\mathrm{XIX}^{\mathrm{e}}$ et $\mathrm{xx}^{\mathrm{e}}$ siècles, la petite ville portuaire, forte de ses fonctions administratives et économiques, s'adapte aux conséquences de " l'accélération simultanée de l'unification nationale, du processus d'industrialisation et de la mondialisation (p. 372) ". Si son port perd en importance à partir du milieu des années 1880, sa population continue 
à croître pour atteindre 6500 habitants en 1930 (chap. 20, Pascal Burguin : "Redon du début du XIX ${ }^{\mathrm{e}}$ siècle à la fin des années trente. Petite ville ou grand port? "). Sur le plan politique, Redon accueille un sous-préfet qui, entre 1848 et 1914, s'affirme comme un personnage clé. De fait, avant la Première Guerre mondiale, à cause de la situation géographique de la ville et de son implantation dans un " petit arrondissement réactionnaire (p. 351) ", le poste n'a rien d'une sinécure. D'ailleurs, sur le long terme, sous l'Empire ou en République, les sous-préfets contribuent par leur action à insérer la ville dans le jeu régional et/ou national (chap. 19 : Jean-François Tanguy, « Les sous-préfets de Redon aux premiers temps du suffrage universel, 18481914 »). Mais il faudra attendre les lois de décentralisation de 1982 pour que la ville tire vraiment profit de sa situation aux confins de trois départements et de deux régions. Ville bleue jusqu'à la Seconde Guerre mondiale, elle passe ensuite à droite. Un temps dans le giron de la gauche suite à la détérioration de sa situation économique (1983-1995), elle échoit au libéral Alain Madelin, par ailleurs conseiller général du canton de Redon, vice-président du conseil régional et ministre de l'économie et des finances (chap. 22 : Jacqueline Sainclivier, "Redon : une ville bleue? »).

Au fil des communications, des documents clés sont reconsidérés et réinterprétés au prisme des recherches actuelles. Déjà publiés et bien connus des historiens et des amateurs éclairés, le cartulaire du IX ${ }^{\mathrm{e}}$ siècle (Cartulaire de l'abbaye Saint-Sauveur de Redon, édition en fac-similé, Rennes, Association des Amis des Archives historiques des diocèses de Rennes, Dol et Saint-Malo, 2 volumes, 1998-2004, 376 p. et 177 p.) et le Manuscrit de la Vilaine du Xvi ${ }^{\mathrm{e}}$ siècle (MAUGER, Michel [dir.], En passant par la Vilaine, de Redon à Rennes, Rennes, Apogée, 1997, 94 p.) sont ainsi présentés au plus grand nombre. Par ailleurs, plusieurs études ouvrent de nouvelles pistes de recherche, dont on ne citera que quelques-unes. Par exemple, Florian Mazel rappelle que l'étude systématique des actes faux ou interpolés du cartulaire reste à faire (p. 101). Dans un autre registre, Philippe Hamon pose au travers du cas redonnais la question des stratégies mises en œuvre par les autorités urbaines en temps de guerre civile : au-delà de l'engagement dans un camp, comment préserver les intérêts d'une ville et de sa population? Quant à Daniel-Odon Hurel, s'appuyant sur la matricule de la congrégation de Saint-Maur, il propose une première prosopographie des Mauristes de Redon. S'interrogeant sur la place de Saint-Sauveur et de ses religieux au sein de la congrégation et sur celle de la prédication de l'abbaye dans la ville, il en appelle au dépassement d'une historiographie vieillie et à une micro-histoire du monastère (p. 217), notamment entre le concile de Trente et l'introduction des Mauristes.

Cette Histoire de Redon prend place avec bonheur dans la bibliothèque d'histoire urbaine publiée aux Presses universitaires de Rennes, aux côtés, par exemple, de l'Histoire de Rennes (AUBERT, Gauthier, CROIX, Alain, DenIs, Michel [dir.], 2006, 296 p.) ou de Villes de Bretagne. Patrimoine et Histoire (AndRIEux, Jean-Yves [dir.], 2014, 383 p.). Comme ces derniers, l'ouvrage est riche d'illustrations de différentes natures (photographies d'édifices et d'objets, cartes, plans, portraits, reproductions de manuscrits) toujours contextualisées et commentées. Même si l'on n'échappe pas à quelques redites - mais n'est-ce pas la loi du genre? - le livre est évidemment précieux pour les Redonnais curieux de l'histoire de leur ville mais aussi stimulant en tant que possible modèle pour d'autres histoires de villes. Une bibliographie sélective permet en outre de compléter les notes accompagnant chacun des articles. Diffusion des derniers acquis de la recherche, ouverture vers d'autres chantiers : le double défi des directeurs de l'ouvrage - clairement formulé dès l'introduction par Daniel Pichot et Georges Provost ("Pour une histoire de Redon ") - est assurément relevé. 